Localized structures in vibrated emulsions

This article has been downloaded from IOPscience. Please scroll down to see the full text article.

2012 EPL 9824002

(http://iopscience.iop.org/0295-5075/98/2/24002)

View the table of contents for this issue, or go to the journal homepage for more

Download details:

IP Address: 141.211.173.82

The article was downloaded on $26 / 06 / 2013$ at $15: 17$

Please note that terms and conditions apply. 


\title{
Localized structures in vibrated emulsions
}

\author{
Claudio Falcón $^{1,2}$, Jake Bruggeman ${ }^{3}$, Matteo Pasquali ${ }^{3}$ and Robert D. Deegan ${ }^{1(a)}$ \\ ${ }^{1}$ Department of Physics and Center for the Study of Complex Systems, Randall Laboratory, University of Michigan \\ Ann Arbor, MI 48109, USA \\ ${ }^{2}$ Departamento de Física, Facultad de Ciencias Físicas y Matemáticas, Universidad de Chile \\ Casilla 487-3, Santiago, Chile \\ ${ }^{3}$ Department of Chemical Engineering, Rice University - 600 Main St., Houston, TX 7ro05, USA
}

received 13 December 2011; accepted in final form 29 March 2012

published online 30 April 2012

PACS 47.57.-s - Complex fluids and colloidal systems

PACS 47.57.Qk - Rheological aspects

PACS 83.60.Rs - Shear rate-dependent structure (shear thinning and shear thickening)

\begin{abstract}
We report our observations of localized structures in a thin layer of an emulsion subjected to vertical oscillations. We observe persistent holes, which are voids that span the layer depth, and kinks, which are fronts between regions with and without fluid. These structures form in response to a finite amplitude perturbation. Combining experimental and rheological measurements, we argue that the ability of these structures to withstand the hydrostatic pressure of the surrounding fluid is due to convection within their rim. For persistent holes the oscillatory component of the convection generates a normal stress which opposes contraction, while for kinks the steady component of the convection generates a shear stress which opposes the hydrostatic stress of the surrounding fluid.
\end{abstract}

Copyright (c) EPLA, 2012

Introduction. - Out-of-equilibrium systems often develop structures that form from the balance of two or more driving mechanisms [1]. A typical example is the Faraday system in which a shallow layer of fluid is vertically vibrated [2]. Above a certain excitation threshold this system displays a wealth of surface wave patterns from highly regular to spatially and temporally chaotic. These patterns emerge from the balance of energy injected by parametric amplification of surface waves with internal dissipation. The robustness of this mechanism accounts for the widespread occurrence of Faraday waves in simple $[1,3]$ and complex [4-6] fluids, and granular materials [7].

As the study and understanding of spatially extended patterns in out-of-equilibrium systems has matured, attention has turned to localized patterns. Unlike in spatially extended patterns in which the domain is the system size and hence known a priori, the central question for localized patterns is: what sets their domain size? There are now many examples of such localized structures in the Faraday system: defects in standing wave patterns $[1,2]$, solitons [8], localized jets [9], oscillons in viscous fluids [10], granular materials [11] and colloidal suspensions [12], to

\footnotetext{
(a) E-mail: rddeegan@umich.edu
}

name a few. Recently, a new class of localized structures — kinks and persistent holes [13-15] — were discovered in the Faraday system with a particulate suspension as the working fluid. These structures are markedly different from the other localized structures: they oscillate about an unstable state and are inaccessible via infinitesimal perturbation from the weakly nonlinear states.

Persistent holes are cylindrical voids which extend through almost the entire layer, they can persist indefinitely, and form only in response to a finite perturbation. Their existence is puzzling because the surrounding fluid ought to flow into to the void due to the hydrostatic pressure difference. Kinks are boundaries that form when the fluid gathers in a subset of the domain; on one side the fluid has a finite depth, and on the other side there is no fluid whatsoever. To date persistent holes and kinks have not been observed in the same system.

The stabilizing mechanism of kinks and persistent holes is currently unknown. Merkt [13] and Ebata et al. [15] argued for shear-thickening, i.e., the increase in viscosity with the increase of the applied shearing stress, as the key factor. However, kinks were also observed in a shearthinning fluid [16], though the presence of a strong yield stress in that system complicates its interpretation. Deegan [17] suggested a different mechanism, independent 
of whether the fluid is shear-thinning or -thickening, based on stress hysteresis. He argued that if the fluid exhibits a hysteretic shear rate response to applied stress, then the net flow in response to the superposition of an oscillatory and a steady stress can be opposite to the direction of the steady stress. In the context of persistent holes, the steady force is generated by the hydrostatic pressure of the surrounding fluid.

In this letter we present an experimental study of vibrated emulsions. We show for the first time that persistent holes and kinks can form in an emulsion, which greatly widens the classes of complex fluid that support persistent holes and establishes that kinks and persistent holes can coexist in a single system. We find that the convection roll identified at a kink in particulate suspensions $[14,17]$ is also present in kinks and persistent holes in emulsions. Furthermore, we show that the stresses generated by this flow can balance the hydrostatic pressure of the surrounding fluid. We find that the convection roll is absent in Newtonian fluids, and thus explaining the stability of these localized structures reduces to identifying the non-Newtonian effect driving the convection roll.

Experiments. - In our experiments a mineral-oil-inglycerol emulsion $6.0 \mathrm{~mm}$ deep was vertically oscillated at a frequency $f$ from 40 to $120 \mathrm{~Hz}$ and at an acceleration $a$ up to $50 \mathrm{~g}$ ( $\mathrm{g}$ being the acceleration of gravity) in a cylindrical container consisting of a plexiglass sidewall (internal diameter $11.2 \mathrm{~cm}$ ) and an aluminum base. The vibration was actuated by an electromechanical shaker (ET-140 from Labworks Inc.) driven with a frequency generator (Agilent 3320A) via a power amplifier (pa-141 from Labworks Inc.). Illumination and acquisition techniques are given in [13]. We used a stable emulsion prepared by dripping paraffin oil (Acros, USA) at $0.3 \mathrm{ml} / \mathrm{min}$ into a continuously stirred premixed solution 2.49 : 100 by weight of dioctyl sulfosuccinate sodium salt (Sigma-Aldrich) in glycerol (Mallinkrodt). Prior to taking any data the freshly prepared emulsion was vibrated at $55 \mathrm{~g}$ and $40 \mathrm{~Hz}$ for $200 \mathrm{~s}$. This step was necessary to eliminate aging of the sample that would otherwise cause irreproducibility. During the experimental runs, our emulsion is stable and no qualitative change is observed. The resulting surfactant-stabilized emulsion had a 0.60 volume fraction of oil and a density $\rho=1020 \mathrm{~kg} / \mathrm{m}^{3}$. The distribution of droplet sizes was determined using optical microscopy and image processing. The average diameter was $3.5 \mu \mathrm{m}$ with a standard deviation of $1.0 \mu \mathrm{m}$.

Phenomenology. - We observed the presence of Faraday waves, kinks, and persistent holes as a function of acceleration and frequency (cf. fig. 2). As acceleration increases at a fixed frequency, the first transition is from a flat surface to a wavy surface; this is the well-known onset of Faraday waves [2]. In this case, the standing wave response is harmonic with respect to the forcing. This feature can be taken as a signature of the non-Newtonian character of the fluid $[6,18]$. The transition to Faraday
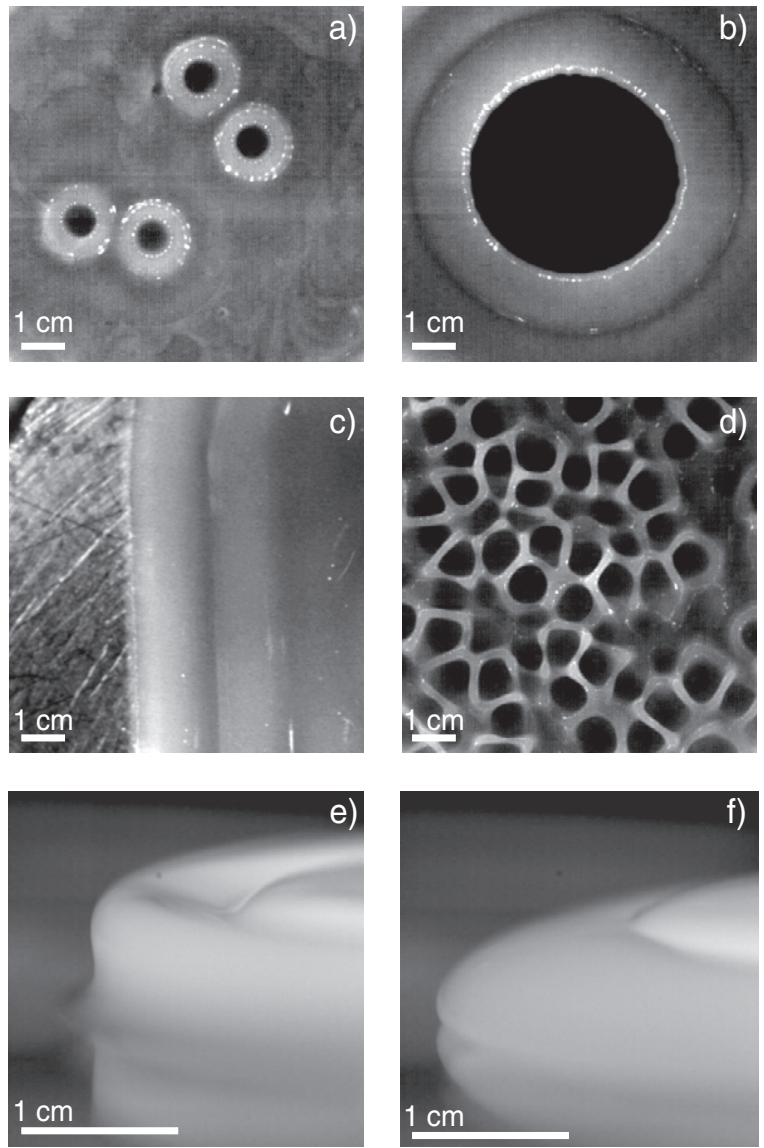

Fig. 1: Localized and extended structures in a $6.0 \mathrm{~mm}$ deep layer of an oil-in-glycerol emulsion vibrated at $f=40 \mathrm{~Hz}$ : (a) holes $(a=49.9 \mathrm{~g})$; (b) circular kink $(a=29.8 \mathrm{~g})$; (c) straight kink $(a=29.8 \mathrm{~g})$; and (d) delocalized state $(a=55.5 \mathrm{~g})$. The gray scale is indicative of the fluid height with white and black corresponding to the highest and lowest points, respectively. In (c) the left side of the image is the bottom of the container. Side view of kink at peak downward (e) and upward (f) acceleration at $f=40 \mathrm{~Hz}$ and $a=29.8 \mathrm{~g}$.

waves is subcritical (the transition threshold changes as we increase or decrease the acceleration) appearing over a narrow range near the transition line (cf. fig. 2). At higher accelerations $(\sim 40 \mathrm{~g})$, persistent holes (cf. fig. 1(a)) form in response to external perturbations of order the layer depth, generated in our experiment by a brief pulse of compressed air directed at the surface $[13,17]$. The formation of a hole is insensitive to the parameters of the air jet, provided the jet is sufficiently strong to push aside fluid over a region of order the layer depth. Below the transition line no persistent holes form regardless of the duration or strength of the air jet.

Persistent holes present themselves to the naked eye as axially symmetric voids $0.8-1.5 \mathrm{~cm}$ in diameter surrounded by a raised torus of fluid (cf. fig. 1(a)). The typical radius of a persistent hole decreases with forcing frequency, from $0.8 \mathrm{~cm}$ at $40 \mathrm{~Hz}$ to $0.4 \mathrm{~cm}$ at $120 \mathrm{~Hz}$, but does not change appreciably with acceleration. Persistent holes 


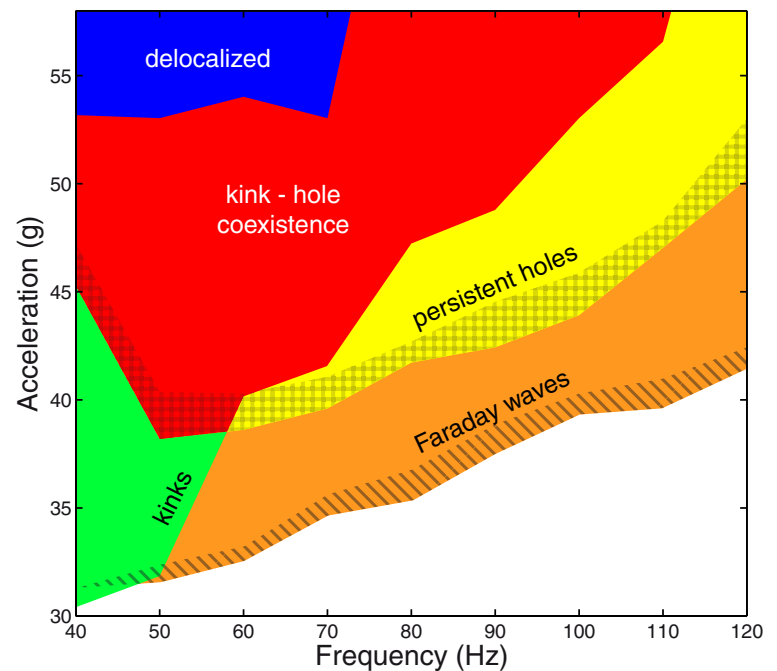

Fig. 2: (Color online) Phase space for a vibrated glycerol/mineral oil emulsion as a function of acceleration $a$ and frequency $f$. The transition from the flat surface to the Faraday wave state is similar to the usual transition in Newtonian fluids except that it is subcritical in the narrow region which smears out the transition point (shown as a hatched region). Persistent holes and kinks manifest throughout the entire experimental range of frequencies in response to a finite amplitude perturbation. The cross-hatched region indicates the metastable regime for persistent holes. The threshold for kinks is lower than that of persistent holes at low frequencies but the converse at high frequencies. In the region labeled kink-hole coexistence both persistent holes and kinks are possible depending on the initial perturbation. In the delocalized regime, seen at low frequencies and large accelerations, the flow is highly erratic as shown in fig. 1(d).

do not move significantly within the layer, nor do they interact with each other provided their rims do not overlap. The transition to persistent holes is preceded by a metastable regime in which persistent holes survive for many thousand of cycles. We define stable persistent holes as those with lifetimes greater than $10^{5}$ cycles. The metastable regime is marked by cross-hatched region in fig. 2 .

The vertical cross-section of persistent holes was deduced by shining a $2.0 \mathrm{~cm}$ wide laser sheet perpendicular to the surface and recording its intersection with the surface using a high-speed camera [13]. Typical profiles at opposite phases of the vibration cycle are shown in fig. 3(a). Persistent holes reach to within $1.0 \mathrm{~mm}$ of the container bottom. The diameter of the hole oscillates harmonically with the forcing frequency. The fluid at the lower part of the hole is approximately static and thus the oscillation results in overhangs during the contraction phase, similar to the one visible on a kink in fig. 1(e).

The fluid in the rim of a persistent hole convects as shown in fig. 3(c). We measured this flow by dispersing fine pepper grains in the emulsion and tracing their paths a)

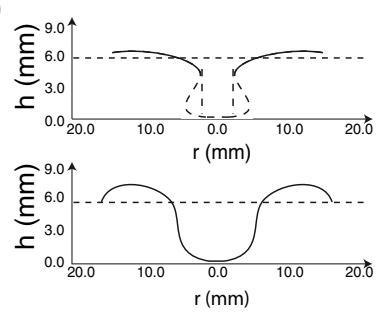

c)

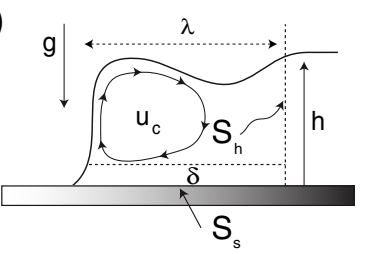

b)

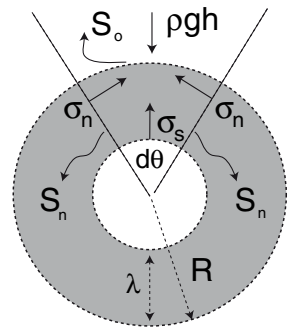

d)

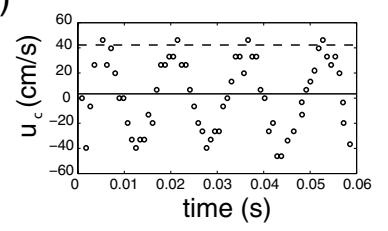

Fig. 3: (a) Oscillating hole profiles at $f=40 \mathrm{~Hz}$ for an acceleration $a=34.5 \mathrm{~g}$. The radius oscillates with the forcing frequency from a low of $\simeq 0.4 \mathrm{~cm}$ to a high of $\simeq 0.8 \mathrm{~cm}$. The dashed part of the curve indicates a lack of data due to the upper part of the hole obscuring the lower part. (b) Stresses acting on a hole. (c) Schematic of a vertical cross-section through a kink or the rim of a hole. (d) Surface velocity $u_{c} v s$. time measured from the particle tracking at $f=60 \mathrm{~Hz}$ and $a=30.6 \mathrm{~g}$ (circles). The solid line corresponds to the mean velocity $u$. The dashed line corresponds to the peak velocity $U$. Note that $u=3.4 \mathrm{~cm} / \mathrm{s}$ is 10 times smaller than $U=42 \mathrm{~cm} / \mathrm{s}$.

on the surface of the hole with a high-speed camera (see supplementary video HoleFlow.avi). This flow consists of a small steady flow $u$ which takes particles along the free surface from the bottom of the rim to the top and back through the bulk superimposed on a larger oscillatory flow with peak speed $U$ that oscillates with the same frequency as the forcing and is directed along the same path as the steady flow. Thus, the speed at any point in the convection roll is $u_{c}=u+U \cos (2 \pi f t)$. The typical speed for the steady and oscillatory flows at the free surface were $2.0-4.0 \mathrm{~mm} / \mathrm{s}$ and $20.0-30.0 \mathrm{~cm} / \mathrm{s}$, respectively. The pepper particles when emerging from the bulk reappeared at least $2.0-2.5 \mathrm{~mm}$ from the bottom of the container; we took this distance as the boundary layer thickness $\delta$. This distance was constant for all $f$ and $a$ within the range of our experiments.

We tried but were unable to recreate the convection roll in Newtonian fluids with viscosities $\eta$ similar to that of the emulsion (glycerol $\eta=8.5 \mathrm{~Pa}$ s and silicone oil $\eta=$ 9.7 Pas) and with the similar driving parameters. Thus, we conclude that the roll is a non-Newtonian effect.

If the applied perturbation forms a sufficiently large void (larger than $2.0 \mathrm{~cm}$ in diameter), the void will continue to grow after the perturbing air jet is shut off. Eventually the growth saturates and the resulting void is either circularly shaped as shown in fig. 1(b) or front-like with a straight edge that runs from one side of the container to the other as shown in fig. 1(c). Even though circularly shaped kinks may look like a big persistent hole (e.g., fig. 1(b)), their 
ability to unroll into a straight front distinguishes them from persistent holes. Irrespectively of their configuration, circular or straight, we call these structures kinks because of the discontinuity in surface height.

In the transition from a flat state to circular kinks, the size of the perturbation matters. Stable kink radii are always larger than $2.0 \mathrm{~cm}$, and thus perturbations with radii less than this will disappear. This feature permits kinks to occur well below the onset of persistent holes at lower frequencies. As in the rim of persistent holes, the fluid within a kink convects (see supplementary video KinkFlow.avi). The flow speed was measured as with the holes, and we obtained $u=2.5-4.0 \mathrm{~cm} / \mathrm{s}$ (note this is an order of magnitude larger than for holes) and $U=$ $22.0-45.0 \mathrm{~cm} / \mathrm{s}$.

Kinks and holes can coexist in the region shown in fig. 2. For example, we can form persistent holes on the fluid side of a kink provided the rim of the hole and the kink are far apart. The formation from the initial flat state of a kink rather than a hole in the coexistence regime depends on the strength of initial perturbation: stronger perturbations are needed to create a kink. A kink can lose stability following a change of the acceleration $a$ or frequency $f$, and shrink; most often it continues to shrink until it vanishes; rarely, the kink shrinks to form a hole; we have never observed the converse.

At high accelerations $(\sim 50 \mathrm{~g})$ and low frequencies $(<70 \mathrm{~Hz})$, we observe a delocalized state analogous to the one observed in particulate suspensions (see fig. 1(d)). A single perturbation nucleates a growing array of persistent holes which ultimately spans the entire domain (see fig. 1(d)). The pattern displays erratic dynamics as the holes merge or move past one another. A Fourier transform of the pattern indicates that the spacing of the persistent holes within the array is similar to the wavelength of underlying Faraday waves.

Rheology. - The convection roll observed in kinks and persistent holes is a non-Newtonian effect, as shown by the inability of a Newtonian fluid to sustain a roll. To determine the stress within persistent holes and kinks, we characterize the emulsion's rheology with a stresscontrolled rheometer (AR-2000ex, TA Instruments) using a cone-plate geometry with an acrylic cone (radius $R_{c}=$ $3.0 \mathrm{~cm}$, angle $\alpha=2^{\circ} 00^{\prime}$ ) at $20^{\circ} \mathrm{C}$. We performed tests with steady shear stress (fig. 4(a), (b) and (c)), oscillatory shear stress in the linear regime (fig. 4(d) and (e)), and oscillatory shear stress in the nonlinear regime (example at $f=1 \mathrm{~Hz}$ and shear stress magnitude of $500 \mathrm{~Pa}$ shown in fig. 4(e)). For the latter, the stress was varied sinusoidally with fixed amplitude and a fixed frequency, the shear rate was recorded, and the inertia of the instrument was subtracted as described in [17]. Sample-to-sample and run-to-run reproducibility was $\pm 10 \%$. Above steady shear rates $\dot{\gamma} \approx 100 \mathrm{~s}^{-1}$ non-rheometric flows developed in the test fixture.
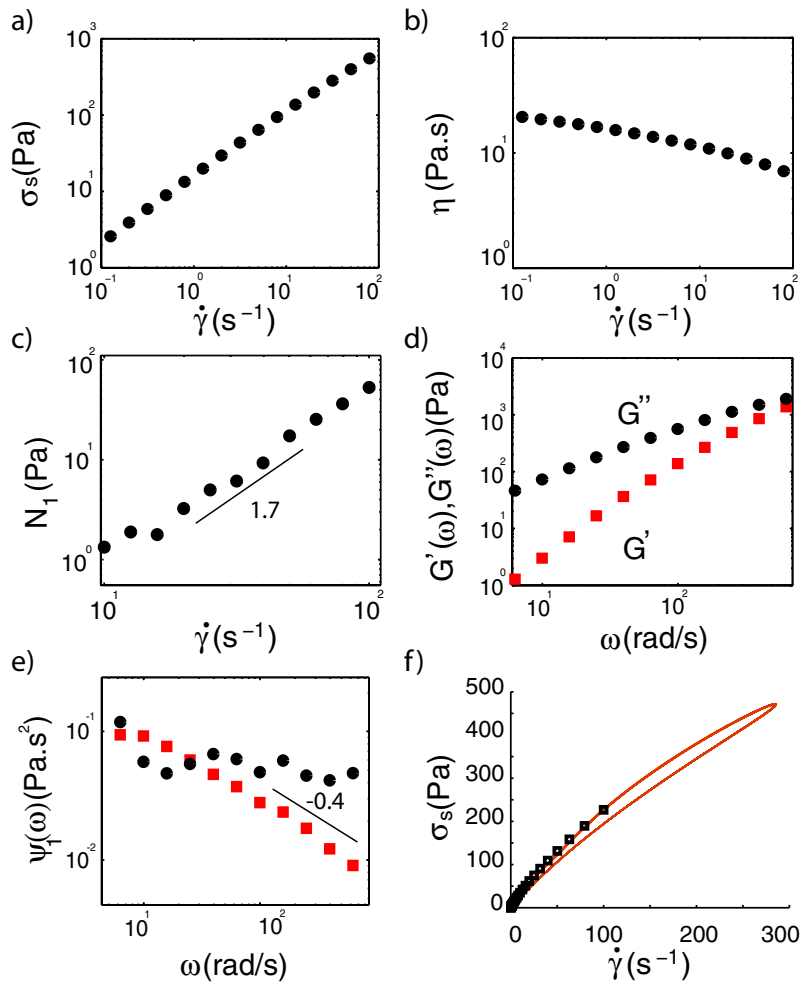

Fig. 4: (Color online) (a) Shear stress $\sigma_{s}$ vs. strain rate $\dot{\gamma}$ from steady measurements. (b) Shear viscosity $\eta$ from steady measurements. (c) Normal stress $\sigma_{n}$ vs. $\dot{\gamma}$ from steady shear measurements. (d) In-phase $G^{\prime}(\omega)(\bullet)$ and out-of-phase $G^{\prime \prime}(\omega)(\square)$ components of the shear modulii as a function of the angular frequency $\omega$. (e) Oscillatory first normal stress coefficient $\psi_{1}(\omega)$ vs. angular frequency $\omega$ in the linear (•) and weakly nonlinear at $\dot{\gamma} \simeq 100 \mathrm{~s}^{-1}$ (口) regimes. (f) Shear stress $\sigma_{s}$ vs. $\dot{\gamma}$ from oscillatory measurements at $f=1 \mathrm{~Hz}$ and shear stress magnitude of $500 \mathrm{~Pa}$. Steady measurements ( are shown for comparison.

The steady shear stress and viscosity measurements (fig. 4(a) and (b)) show that the emulsion is shearthinning, which is the expected behavior of surfactant stabilized emulsions [19-21]. The yield stress — if present at all- is weaker than $0.3 \mathrm{~Pa}$. The first normal stress difference $N_{1}$ follows a power law in $\dot{\gamma}^{\alpha}$, with $\alpha \sim 1.7$, which given our limited range of $\dot{\gamma}$ is consistent with the expected $\dot{\gamma}^{2}$ behavior. As shown in fig. 4(d), the oscillatory measurements in the linear regime show that the out-of-phase component of the shear modulus $G^{\prime \prime}(\omega)$ is 10 times larger than the in-phase component $G^{\prime}(\omega)$ as a function of $\omega$, indicating that the response is primarily viscous for low frequencies [20,21]. We computed the oscillatory first normal stress coefficient $\psi_{1}(\omega)=N_{1}(\omega) / \dot{\gamma}(\omega)^{2}$ as shown in fig. $4(\mathrm{~d})$, where $N_{1}(\omega)$ and $\dot{\gamma}(\omega)$ are the normal stress and shear rate Fourier amplitudes at angular frequency $\omega$. In the linear regime, $\psi_{1}(\omega)$ is roughly constant. At oscillatory stress amplitudes exceeding $\sigma_{o}=200 \mathrm{~Pa}$, the response becomes hysteretic and weakly nonlinear (cubic nonlinearities are less than 
$5 \%$ of the linear response for stress amplitudes between 200 and $600 \mathrm{~Pa}$ ) as is shown in fig. 4(f). This is observed also in fig. 4(e) as $\psi_{1}(\omega)$ decreases with increasing $\omega$ when the stress amplitude is larger than $\sigma_{o}$.

Besides the direct measurement of $N_{1}$, by partially filling the measurement geometry we can estimate the second normal stress difference $N_{2}$ for a given $\dot{\gamma}$ following the procedure described in $[22,23]$. We performed this procedure for $\dot{\gamma}$ between 10 and $100 \mathrm{~s}^{-1}$ (not shown here), which we can use to characterize all the normal stress differences in our emulsion.

Force balance. - We now show that the measured stress response of the fluid is sufficient to account for the ability of persistent holes and kinks to withstand the hydrostatic pressure of the surrounding fluid. We calculate the force on a stationary convection roll in a kink by integrating the stress tensor over the surfaces surrounding the kink: $S_{h}, S_{s}$, and the free surface (see fig. $3(\mathrm{c})$ ). $S_{h}$ is acted on by the hydrostatic pressure of the surrounding fluid, $S_{s}$ is acted on by the drag from container bottom and the free-surface is stress free. The forces per unit length acting on the kink are thus $F_{p} \simeq \frac{1}{2} \rho a h^{2}$ (from the hydrostatic term) and $F_{\eta} \simeq \sigma_{s} \lambda$ (from the drag term). The force per unit length coming from surface tension is negligible, as it is estimated to be 10 times smaller than both contributions. The geometrical parameters are shown in fig. 3(c): $h$ is the layer depth behind the kink, which is higher than the initial layer depth due to volume conservation, and $\lambda$ is the kink's width. $\sigma_{s}$ is the shear stress at steady shear rate $\dot{\gamma}$. In what follows, we use our rheological measurements to estimate the stresses produced by the flow.

For a stationary kink we can use a simple block model as the one described in $[14,17]$ where a kink can be thought as a two dimensional structure that extends infinitely out of the page (see fig. 3(c)). We approximate the shear rate $\dot{\gamma} \approx u_{c} / \delta$ where $\delta$ is the boundary layer thickness. We assume that to leading order the average over one oscillation yields the average of the terms: $\overline{a h^{2}}=\bar{a} \bar{h}^{2}$ and $\overline{\sigma_{s} \lambda}=\sigma_{s}(\overline{\dot{\gamma}}) \bar{\lambda}$, where $\overline{\text { connotes }}$ the average. Averaging over one oscillation cycle of $\bar{a}=g$ and $\overline{\dot{\gamma}}=u / \delta$. Measurements at $f=40 \mathrm{~Hz}$ and $a=30.6 \mathrm{~g}$ yield $h=7.5 \mathrm{~mm}, \lambda=7.0 \mathrm{~mm}, u=35.0 \mathrm{~mm} / \mathrm{s}, \delta=2.5 \mathrm{~mm}$. Thus, $\dot{\gamma}=14 \mathrm{~s}^{-1}$ and $\sigma_{s}=44 \mathrm{~Pa}$ at that shear rate, $F_{\eta} \approx$ $308 \mathrm{mN} / \mathrm{m}$, and $F_{p} \approx 275 \mathrm{mN} / \mathrm{m}$. Hence, the reaction force on the convection roll is the right order of magnitude to balance the hydrostatic pressure of the surrounding fluid. This near equality holds for all frequencies and accelerations along the transition line to kinks in fig. 2 .

The foregoing force balance calculation fails for persistent holes because the steady flow velocity is about ten times smaller than in a kink, and thus the mechanism sustaining persistent holes must be different. Our results, as we now show, suggest that a hoop stress, similar in spirit to that in the rod-climbing effect, provides the force needed to resist the hydrostatic pressure of the surrounding fluid. Consider an angular wedge from the torus of fluid convecting in the rim (see fig. 3(b)). The normal stress component acting in the direction tangent to the center line of the torus pushes on the sides of the wedge (labeled $S_{n}$ ) resulting in a net force per unit angle $\mathcal{F}_{N}=\sigma_{n} \lambda h$ pushing outwards. The hydrostatic force pushing inwards per unit angle is $\mathcal{F}_{p}=\frac{1}{2} \rho g h^{2} R$, from the integration of the hydrostatic pressure over the angular section of the outer surface (labeled $S_{o}$ ). As with the kinks, we average over a cycle. The oscillatory contribution to the hydrostatic pressure vanishes, but the normal stress is positive regardless of the flow direction and therefore averaging picks up contributions from both the steady and oscillatory shear rate. The latter is dominant because $u \ll U$ and $\dot{\gamma} \approx U / \delta=80-100 \mathrm{~s}^{-1}$.

Using the conventional nomenclature for normal stresses directions, we designate the radial direction in the plane of circulation of the roll to be the first direction, the circulation direction of the roll to be the second direction, and the direction perpendicular to plane of circulation to be the third direction. The corresponding normal stress differences are $N_{1}, N_{2}$, and $N_{3}$ where $N_{3}=-\left(N_{1}+\right.$ $N_{2}$ ). The hoop stress arises from the third component of the normal stress, and thus $\sigma_{n}=N_{1}+N_{2}$. At $f=$ $40 \mathrm{~Hz}, a=32.3 \mathrm{~g}$ and $\dot{\gamma} \simeq 100 \mathrm{~s}^{-1}, h=6.0 \mathrm{~mm}, \lambda=7.0 \mathrm{~mm}$, $R=18.0 \mathrm{~mm}$. Our rheological measurements give $N_{1} \simeq$ $200 \mathrm{~Pa}$ in the linear regime and $60 \mathrm{~Pa}$ in the weakly nonlinear regime at $\dot{\gamma} \simeq 100 \mathrm{~s}^{-1}$ (evaluating $\psi_{1}(\omega)$ at $\omega=2 \pi f$, using the estimated amplitude of the oscillatory shear rate and averaging over a cycle) and $N_{2}=$ $8 \mathrm{~Pa}$. Thus, $\mathcal{F}_{N} \simeq 2.5-8.2 \mathrm{mN}$ (using linear and weakly nonlinear measurements as estimation bounds) and $\mathcal{F}_{p} \simeq$ $3.2 \mathrm{mN}$. Hence, the normal stress is of the right order of magnitude to oppose the hydrostatic pressure from the surrounding fluid that would otherwise collapse the hole. These results suggest that the normal stresses stabilize holes.

We also tested an attractive emulsion, selected because it exhibits a negative normal stress [24] and would provide a critical test of the importance of normal stresses. This oil-in-water emulsion was prepared by dripping mineral oil (Acros) into a premixed solution of water and a nonionic surfactant, Span 80 (Sigma-Aldrich), in proportion such that the weight of the surfactant equaled $5 \%$ of the final weight of the emulsion. Various volume fractions of oil were tested ranging from 0.2 to 0.8 . We characterized the emulsion's rheology with a strain-controlled rheometer (ARES-LS1, TA Instruments) using a steel plateplate geometry (diameter $\phi=2.5 \mathrm{~cm}$, separation gap $d=$ $1.00 \mathrm{~mm}$ ) at $20^{\circ} \mathrm{C}$. Figure 5 shows the steady measurements of shear viscosity $\eta$ and the magnitude of the normal stress $\left|N_{1}\right|$ as a function of shear stress $\dot{\gamma}$ for the $60 \%$. This emulsion is shear-thinning, and in the shear rate range $\dot{\gamma} \sim 1-10 \mathrm{~s}^{-1}$ the normal stress is negative. We were able to form kinks in these samples, but not persistent holes. The absence of the latter is consistent with our argument 


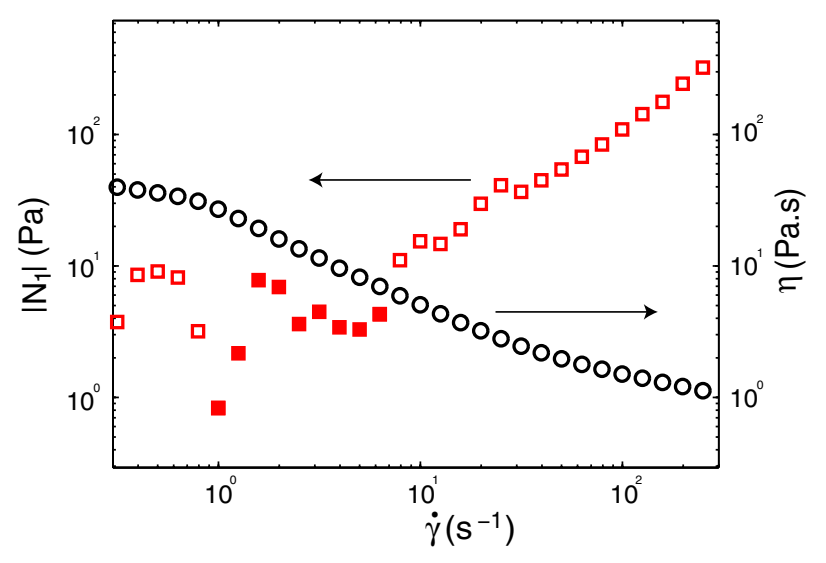

Fig. 5: (Color online) Shear viscosity $\eta(\circ)$ and magnitude of the normal stress $(\square)$ vs. $\dot{\gamma}$ for an attractive water/oil emulsion (0.6 oil concentration). Filled symbols indicate negative $N_{1}$.

that normal forces are essential for sustaining persistent holes.

Discussion and conclusions. - We find that a vertically vibrated shear-thinning emulsion can sustain kinks and holes. Thus, shear-thickening is unnecessary for these structure to form contrary to previous claims [13]. Furthermore, we show through order-of-magnitude calculations that these structures are stabilized by stresses arising from convection localized within a kink or the rim of a persistent hole. In the case of kinks, we argued that the drag on the container's base caused by the convection opposes the hydrostatic stress of the surrounding fluid. The rectified flow in the roll is crucial for sustaining this stress, but we do not currently understand its origin. One plausible mechanism, suggested by the observation of stress hysteresis in our emulsion, is the model proposed by Deegan [17] in which stress hysteresis produces an average displacement opposite to the average stress.

In the case of persistent holes, we argued that the normal stress generated by the oscillatory flow in the convection roll produces a hoop stress which opposes contraction of the rim. This effect is analogous to the rod climbing effect [20]. The back-and-forth motion of the rim in response to the applied sinusoidal acceleration appears to be sufficient to generate the shearing responsible for the normal stress.

The presence of kinks and persistent holes has now been observed in particulate suspensions, viscoplastic fluids and now emulsion. This growing list of examples and the simplicity of the mechanisms we invoked to motivate their stability suggests that kinks and persistent holes are generic to complex fluids subjected to vertical vibrations.
The authors would like to thank R. G. LARSON and P. DEsAI. This work was supported by the National Science Foundation under Grant No. 0932600051015. CF acknowledges FONDECYT under grant 11090449. RDD thanks the James S. McDonnell Foundation for support through a 21st Century Science Initiative in Studying Complex Systems-Research Award.

\section{REFERENCES}

[1] Cross M. C. and Hohenberg P. C., Rev. Mod. Phys., 65 (1993) 851.

[2] Faraday M., Philos. Trans. R. Soc. London, 121 (1831) 299.

[3] Edwards W. S. and Fauve S., J. Fluid Mech., 278 (1994) 278.

[4] Raynal F., Kumar S. and Fauve S., Eur. Phys. J. B, 9 (1999) 175.

[5] Ballesta P. and Manneville S., J. Non-Newtonian Fluid Mech., 147 (2007) 23.

[6] Wagner C., Müller H. W. and Knorr K., Phys. Rev. Lett., 83 (1998) 208.

[7] Aranson I. and Tsimring L., Rev. Mod. Phys., 78 (2006) 641.

[8] Lioubashevski O., Arbell H. and Fineberg J., Phys. Rev. Lett., 76 (1996) 3859.

[9] Longuet-Higgins M. S., J. Fluid Mech., 127 (1983) 103; Zeff B. W., Fineberg J. and Lathrop D. P., Nature, 403 (2000) 401.

[10] Arbell H. and Fineberg J., Phys. Rev. Lett., 85 (2000) 756.

[11] Umbanhowar P. B., Melo F. and Swinney H. L., Nature, 382 (1996) 793.

[12] Lioubashevski O., Hamiel Y., Agnon A. and Reches Z., Phys. Rev. Lett., 83 (1999) 3190.

[13] Merkt F. S. et al., Phys. Rev. Lett., 92 (2004) 184501.

[14] Ebata H., Tatsumi S. and Sano M., Phys. Rev. E, 79 (2009) 066308.

[15] Ebata H. and Sano M., Phys. Rev. Lett., 107 (2011) 088301.

[16] Shiba H. et al., Phys. Rev. Lett., 98 (2007) 044501.

[17] Deegan R. D., Phys. Rev. E, 81 (2010) 036319.

[18] Ballesta P., Lettinga M. P. and Manneville S., Soft Matter, 7 (2011) 11440.

[19] Pal R., Curr. Opin. Colloid Interface Sci., 16 (2011) 41.

[20] Macosko C. W., Rheology, Principles, Measurements and Applications (Wiley-VHC) 1994.

[21] Larson R., The Structure and Rheology of Complex Fluids (Oxford University Press) 1998.

[22] Alcoultlabi M. et al., Rheol. Acta, 48 (2009) 191.

[23] Bird R. B., Armstrong R. C. and Hassager O., Dynamics of Polymeric Liquids (Wiley) 1987.

[24] Montesi A., Peña A. A. and Pasquali M., Phys. Rev. Lett., 92 (2004) 058303. 